Literature Cited

1) Batchelor, G. K.: Proc. Camb. Phil. Soc., 43, 533 (1947) 2) Bobkowicz, J. A. and W. H. Gauvin: Chem. Eng. Sci., 22, 229 (1967)

3) Cengel, J. A., A. A. Faruqui, J. W. Finnigan, C. H. Wright and J. G. Knudsen: A.I.Ch.E.J., 8, 335 (1962)

4) Chuang, H. and J. E. Cermak: ibid., 13, 266 (1967)

5) Elenkov, D.: Collect. Czech. Chem. Commun., 31, 4072
(1966)

6) Hinze, J. O.: A.I.Ch.E.J., 1, 289 (1955)

7) Idem: "Turbulence"' McGraw-Hill, New York (1959)

8) Kolmogoroff, A. N.: Compt. Rend. Acad. Sci., URSS, 30, $301(1941)$

9) Kuboi, R., I. Komasawa and T. Otake: Kagaku Kōgaku, 36, 989 (1972)

10) Laufer, J.: N.A.C.A.Tech. Repts., 1174 (1954)

11) Levich, V. G.: "Physicochemical Hydrodynamics", Prentice-Hall (1962)

12) Martin, G. Q. and L. N. Johanson: A.I.Ch.E.J., 11, 29 (1965)

13) Rietema, K.: Advances in Chem. Eng., 5, 278 (1964)

14) Rushton, J. H., E. W. Costich, and H. J. Everett: Chem. Eng. Prog., 46, 467 (1950)

15) Schwartzberg, H. G. and R. E. Treybal: Ind. Eng. Chem., Fundamentals, 7, 1 (1968)

16) Idem: ibid., 7, 6 (1968)

\title{
RECIRCULATION AND TURBULENCE BY JET FLOW CONFINED IN VESSEL*
}

\author{
MASAYOSHI SADAKATA AND DAIZO KUNII \\ Department of Chemical Engineering, University of Tokyo, Tokyo, Japan
}

\begin{abstract}
Recirculation and the turbulence characteristics by jet flow confined in vessel were studied experimentally to examine Curtet-Craya's theory.

The location of recirculation zone was first measured visually. The starting position of recirculation zone was well explained by Curtet-Craya's theory.

Measurement of turbulence characteristics in the recirculation zone was carried out by hot-wire anemometer. These results show that increase in turbulence and high anisotropy of turbulence are caused by the low-frequency turbulence newly produced in the recirculation zone. Curtet-Craya's assumption that turbulence is isotropic seems not to be appropriate in the recirculation zone. Turbulence intensities at the position of maximum recirculation flow correlated with Curtet number.
\end{abstract}

\section{Introduction}

Many experimental or theoretical investigations of fluid mixing have been reported, but most of them are not based on the fluid mechanics of flows in a vessel.

Provided the flow characteristics, especially rate of recirculation and turbulence intensity, can be estimated, it becomes possible to predict the macromixing characteristics of the flow on the basis of the above quantities.

From the above point of view, turbulent jet stream was selected to induce recirculation and turbulence in a vessel, and the flow dynamics of the fluid flow was investigated experimentally.

\section{* Received on December 11, 1971}

Presented at the 35th Annual Meeting (April 1970), 4th Autumn Meeting (Oct. 1970) and 36th Annual Meeting (April 1971) of the Soc. of Chem Engrs., Japan.
This flow system is familiar, because it resembles that of burners applied in common furnaces, as shown schematically in Fig. 1.

When the ratio of jet flow to ambient flow increases to some critical value, recirculation flow occurs, and eddies like Karman vortex are observed between the jet flow and the recirculation flow.

Curtet \& Craya $^{5)}$ solved this flow system analytically, and derived the similitude parameter $C_{u}$ called the Curtet number:

$$
C_{u}=-\frac{3}{2} R_{0}^{2}+R_{0}+\frac{R_{0}^{2}}{\lambda_{0}}
$$

$R_{0}=$ (jet flow rate)/(total flow rate)

$\lambda_{0}=($ jet nozzle diameter $) /$ (duct width)

According to their theory, ratio of recirculation flow, location of recirculation zone and effective width of jet can be determined approximately by the above simple parameter $C_{u}$. But the ratio of recirculation flow rate derived from their theory diverges to infinitely large value at some point downstream from 


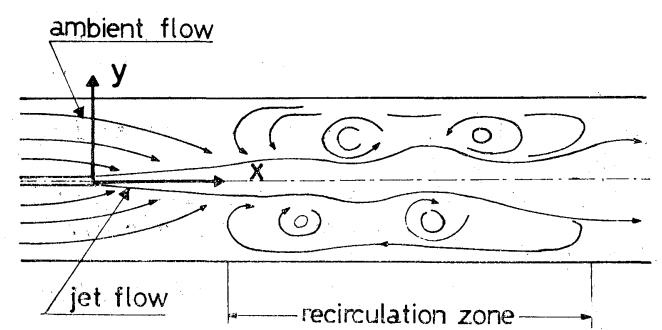

Fig. 1 Jet stream in flow with recirulation

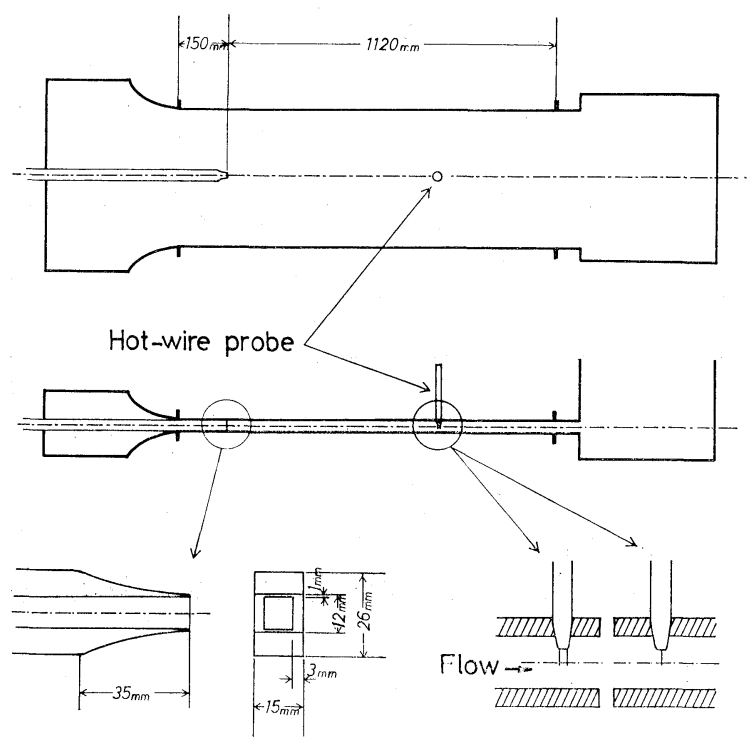

Details of the jet nozzle

Fig. 2 Experimental apparatus

the jet. This contradicts the real phenomena. The above contradiction originates in their assumption, namely that the turbulence in the recirculation zone is isotropic.

In the present work, position of the recirculation zone was first measured to compare with CurtetCraya's theory, and then the turbulence characteristics in the recirculation zone were observed experimentally.

\section{Experimental Apparatus and Methods}

Fig. 2 shows an experimental apparatus designed for two-dimensional water-water system. Length of the vessel was $1120 \mathrm{~mm}$ from the tip of jet nozzle. Width of the vessel was changeable from $140 \mathrm{~mm}$ to $260 \mathrm{~mm}$, keeping the inside thickness of $15 \mathrm{~mm}$. Convergent entrance was designed to make the ambient flow parallel to the jet stream from the nozzle, the contraction ratio being $1 / 20$. The jet flow rate was, adjusted from 0 to $40 \mathrm{l} / \mathrm{min}\left(R_{e d}=6.7 \times 10^{4}\right)$, and the ambient flow rate from 0 to $130 \mathrm{l} / \mathrm{min}$.

To make the flow visible and to measure the recirculation zone, both pulverized aluminum flake and black ink were applied as the tracer.
Measurement of turbulence was made by hotwire anemometer using the constant-temperature method: A hot-wire of diameter $10 \mu$ and length $1.4 \mathrm{~mm}$ was used. This hot-wire was made of tungsten and plated with gold to preserve it from corrosion. The hot-wire probe was set up as shown in Fig. 2. For measurement of $u$, the hot-wire was set parallel to the $y$-direction and for measurement of $v$, it was set parallel to the $x$-direction. The relation between voltage signal and velocity was linearized by the linearizer. A real-time digital correlator and a spectrum analyzer were used to analyze velocity fluctuation.

\section{Results and Discussion}

\section{Location of recirculation zone}

The position where recirculation flow started was measured visually. Fig. 4 shows the distance of the above starting position in the $140 \mathrm{~mm}$-wide duct, to that where the jet flow rate was varied. Similar data are shown in Fig. 5 for the vessel width $260 \mathrm{~mm}$. From these results, this position was found to move toward upstream with increasing rate of jet stream. Absolute value of jet flow rate and ambient flow rate do not seem to affect the location of the starting position, as long as the ratio of jet flow rate to ambient flow is kept constant.

Let us compare these results with Curtet-Craya's theory. Starting position of recirculation is determined theoretically on the basis of Curtet's theory as follows. From the Navier-Stokes Eqs., the following Eqs. are derived under certain assumptions' (see Appendix A).

1) Eq. of motion of ambient flow

$$
\frac{1}{2} \rho \bar{U}_{a}^{2}+P_{a}=P_{t}=\text { const. }
$$

2) Eq. of motion of jet

$$
\bar{U}_{0} \frac{\partial \bar{U}_{0}}{\partial x}+\frac{\partial\left(\bar{u}^{2}-\bar{v}^{2}\right)}{\partial x}=-\left.\frac{\partial \bar{u} \bar{v}}{\partial y}\right|_{y=0}-\frac{1}{\rho} \frac{\partial \bar{P}_{a}}{\partial x}
$$

3) Eq. of total momentum flux

$$
\int_{-h}^{h}\left[\bar{U}^{2}+\left(\bar{u}^{2}-\bar{v}^{2}\right)\right] d y+\frac{2 h}{\rho} \bar{P}_{a}=\frac{M}{\rho}=\text { const. }
$$

4) Eq. of total mass flux

$$
\int_{-h}^{h} \bar{U} d y=Q=\text { const. }
$$

The mean. velocity distribution and Reynolds stress are assumed as

$$
\begin{array}{rlr}
x=0: & \bar{U}=\bar{U}_{a} \quad|y|>l \\
& \bar{U}=\bar{W}_{0}+\bar{U}_{a}:|y| \leqq l \\
x>0: & \bar{U}=\bar{W}_{0} \exp -\frac{\pi}{4}\left(\frac{y}{l}\right)^{2}+\bar{U}_{a} \text { (see Fig. 3) } \\
\overline{u v}=-\varepsilon \frac{\partial \bar{U}}{\partial y}=-2 \sigma \bar{W}_{0} l \frac{\partial \bar{U}}{\partial y} \quad \text { (see Appendix B) }
\end{array}
$$

where

$$
l \text { (effective width of jet })=\frac{1}{W_{0}} \int_{0}^{h} \bar{W} d y
$$

Turbulence is assumed to be isotropic as

$$
\bar{u}^{2}=\bar{v}^{2}
$$




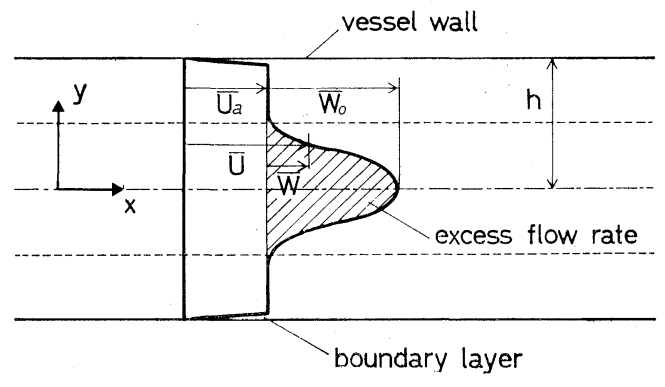

Fig. 3 Two-dimensional jet confined in vessel

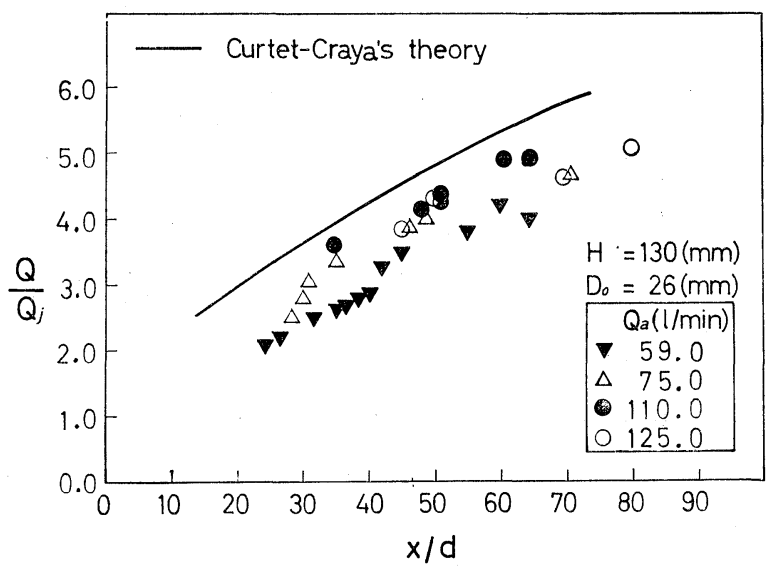

Fig. 5 Starting position of recirculation flow

Substituting these expressions into Eqs. (1), (2), (3) and (4), and introducing dimensionless variables as follows;

$$
R=\frac{q}{Q} \quad \lambda=\frac{l}{h} \quad X=\frac{\sigma x}{h}
$$

where

$$
q \text { (excess flow rate) }=2 \bar{W}_{0} l
$$

One gets from Eqs.(1), (2) and (4)

$$
\frac{\partial}{\partial x}\left(\frac{R^{2}}{2 \lambda^{2}}+\frac{R-R^{2}}{\lambda}\right)=-\pi \frac{R^{2}}{\lambda^{3}}
$$

from Eqs. (1), (3) and (4)

$$
-\frac{3}{2} R^{2}+R+K \frac{R^{2}}{\lambda}=\frac{2 h}{\rho Q^{2}}\left(M-2 h \bar{P}_{t}\right)-\frac{1}{2}=C_{u}
$$

where

$$
K=\int_{0}^{h / l}\left(\frac{\bar{W}}{\bar{W}_{0}}\right)^{2} d\left(\frac{y}{l}\right)
$$

Therefore,

$$
\begin{array}{lll}
K=1 & \text { at } & X=0 \\
K \fallingdotseq 0.707 & \text { at } & X>0
\end{array}
$$

The change of $R, \lambda$ along the $X$-axis can be easily calculated by the Runge-Kutta-Gill method. The position of $R=1$ is the starting position of recirculation (see Appendix C).

Calculated line was added to Fig. 4 and Fig. 5, suggesting the adequency of the above theoretical calculation to explain the tendency of experimental results. Precisely speaking, however, the calculated

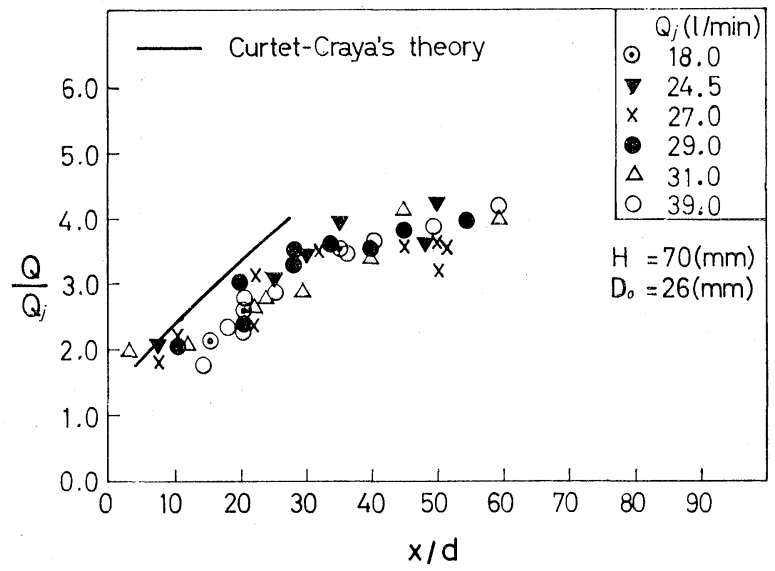

Fig. 4 Starting position of recirculation flow

line was located on the upstream side compared with the experimental data. This discrepancy originates in the difference of definitions for the starting position. The theoretical definition is the position where ambient flow velocity becomes zero. On the other hand, the experimental one was defined as the position where the recirculation flow clearly started. In practical experiments, there was a zone where the water was at rest between the above two positions. Therefore, it might be considered to have the theoretical line on the up-stream side compared with the experimental data.

\section{Gharacteristics of turbulence}

Measured distribution of turbulence intensity is shown in Fig. 6 for flow having recirculation. This result seem to be clearly different from the result for free jet streams already investigated by many researchers (see Ref. 12). In case of free jet $u^{\prime} \mid \bar{U}$ (the relative turbulence intensity) is nearly constant regardless of the distance from the tip of jet nozzle. But when recirculation flow occurs, the relative turbulence intensity increases in the recirculation zone. This means that new energy-containing eddies were being produced in the recirculation zone. This turbulence newly produced in the recirculation zone may be called recirculation turbulence.

Fig. 7 shows the ratio of $x$-direction turbulenceintensity to $y$-direction turbulence-intensity in flow without recirculation, where anisotropy of turbulence is observed only on the center line of jet. Turbulence in the other part of the flow can be assumed to be nearly isotropic. When recirculation occurred, the ratio of turbulence-intensities varied very much, as shown in Fig. 8. It was found that the turbulence became highly anisotropic in flow accompanied by recirculation. Therefore, Curtet-Craya's assumption that the turbulence is isotropic seems not to be appropriate in the recirculation zone. This result suggests that, although Curtet-Craya's theory well describes the fluid dynamics of the upstream region of the recirculation zone where turbulence is nearly isotropic, and could well explain the tendency of the starting 


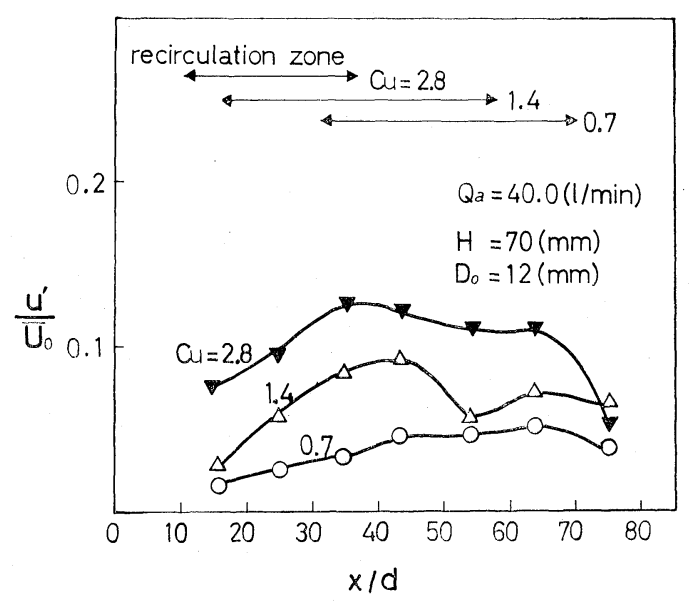

Fig. 6 Distribution of relative turbulence intensities along the center line of jet stream

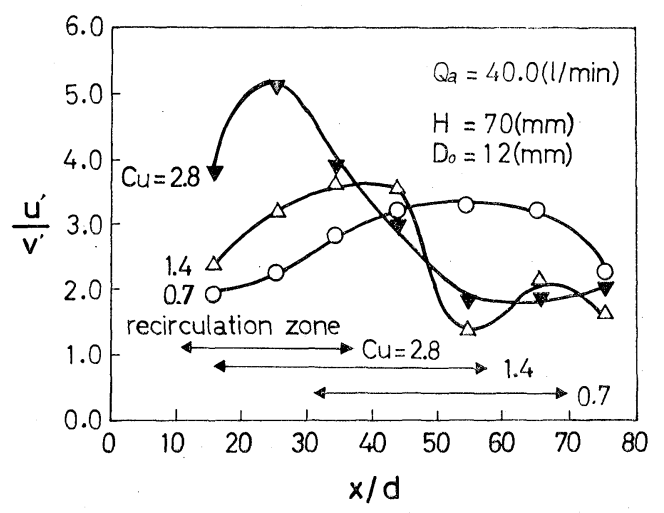

Fig. 8 Ratio of turbulence intensities along the center line of jet stream

position of the recirculation zone, it is unable to describe the fluid dynamics in the recirculation zone where turbulence becomes highly anisotropic.

Measurement of the energy spectrum of velocity fluctuation was made when recirculation flow started at the point $x / d=19.0$. Fig. 9 shows the energy spectrum measured at the point $x / d=15.9$ where recirculation flow did not occur, and Fig. 10 illustrates the spectrum at the point $x / d=45.0$ in the recirculation zone. From Fig. 9, it can be observed that turbulence energy was contained in two kinds of eddies, namely higher-frequency side eddies and lower-frequency side eddies. In Fig. 10, higher-frequency side eddies disappeared and only lower-frequency side eddies were observed. This result indicates that turbulence newly produced in the recirculation zone was lowfrequency eddies. High anisotropy of turbulence in the recirculation zone might be caused by this low-frequency turbulence newly produced in the recirculation zone.

Next, the relation between turbulence-intensity in the recirculation zone and Curtet number was investigated experimentally. Fig. 11 shows the laterally averaged turbulence intensities at the point

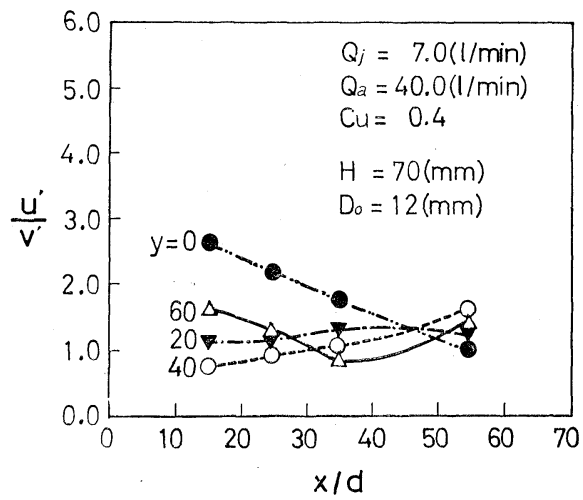

Fig. 7 Ratio of turbulence intensities

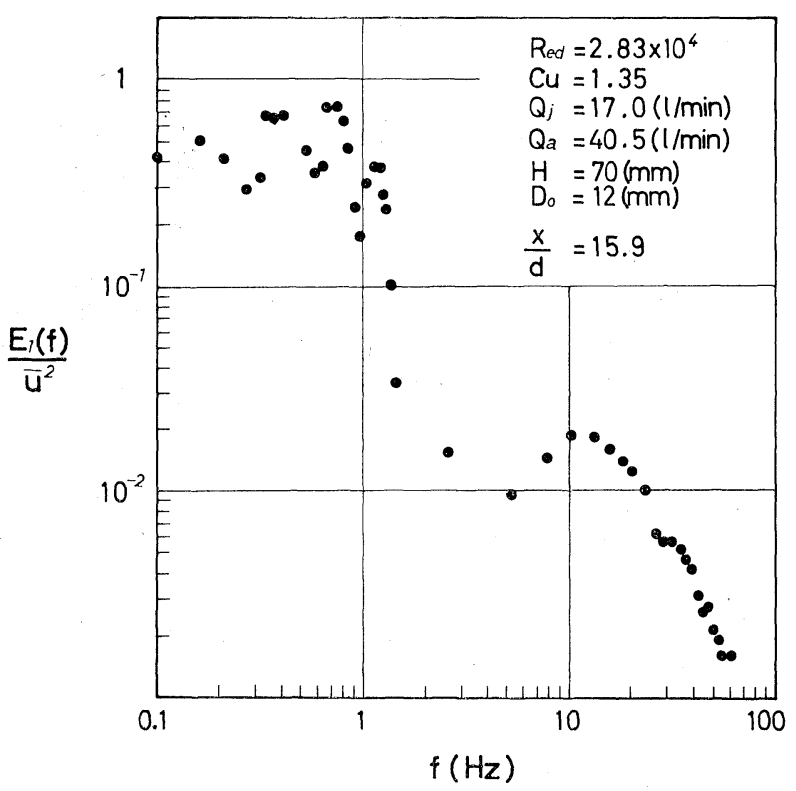

Fig. 9 Energy spectrum of turbulence velocity $u$ at $y / d=0$

of maximum recirculation flow rate vs. Curtet munber, the jet flow rate being taken as a parameter. From the above results, it was found that the intensity of the turbulence was clearly affected by Curtet number. According to Curtet-Craya's theory, the change of $C_{u}$ means the change of recirculation flow rate and the position of recirculation. The recirculation flow rate increased with increasing Curtet number. Thus the flow pattern of recirculation zone seemed closely related to the intensity of recirculation turbulence. This is important in connection with the possibility of controlling the turbulence intensity by adjusting the entrance flow condition, namely the ratio of jet flow rate to ambient flow rate, as well as the geometry of the vessel, i.e. the ratio of jet nozzle to vessel width.

The authors derived theoretical equations to explain the phenomena reported in the present paper more precisely, based upon the hydrodynamic way of thinking. Comparison of the calculation with 


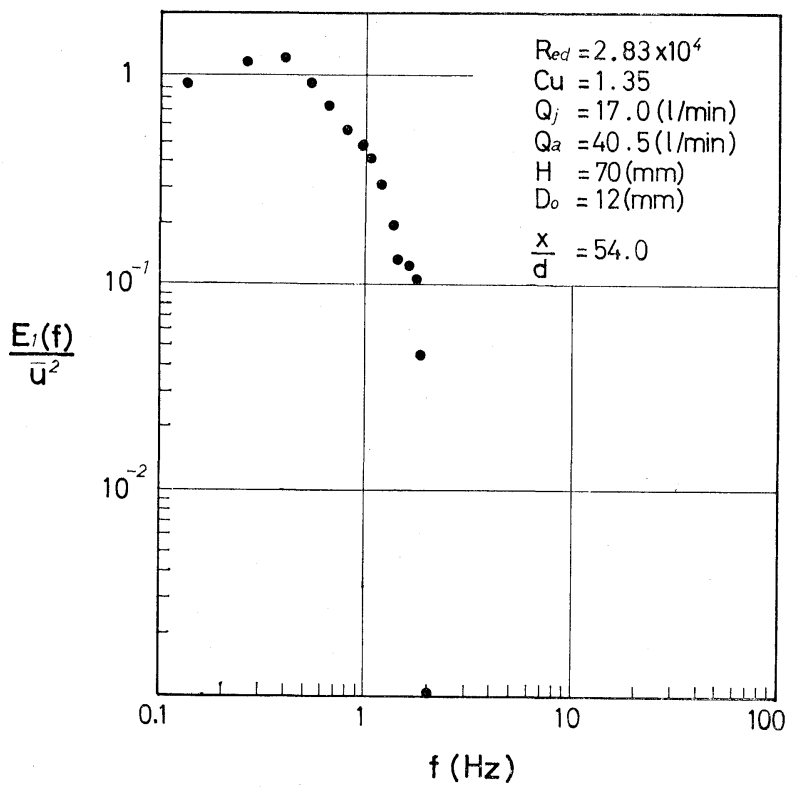

Fig. 10 Energy spectrum of turbulence velocity $u$ at $y / d=0$

the experimental data shall be reported elsewhere.

\section{Conclusion}

1) Starting position of recirculation was well explained by Curtet-Craya's theory.

2) Turbulence intensity was larger in flows with recirculation zone compared with flows without recirculation, and its values at the position of maximum recirculation flow rate were correlated with Curtet number.

3) Highly anisotropic turbulence was observed in the recirculation zone. Curtet-Craya's assumption that the turbulence is isotropic seemed not to be appropriate in the recirculation zone.

4) Low-frequency turbulence was produced in the recirculation zone. Increase in turbulence intensity and anisotropy of turbulence were caused by low-frequency turbulence newly produced in the recirculation zone.

\section{Appendix}

\section{(A) Derivation of basic equations}

The Reynolds equation of motion for two-dimensional incompressible turbulent flow can be obtained from the Navier-Stokes equation.

$$
\begin{aligned}
& \bar{U} \frac{\partial \bar{U}}{\partial x}+\bar{V} \frac{\partial \bar{U}}{\partial y}+\frac{\partial \bar{u}^{2}}{\partial x}+\frac{\partial \overline{u v}}{\partial y}=-\frac{1}{\rho} \frac{\partial \bar{P}}{\partial x}+\nu\left(\frac{\partial^{2} \bar{U}}{\partial x^{2}}+\frac{\partial^{2} \bar{U}}{\partial y^{2}}\right) \\
& \bar{U} \frac{\partial \bar{V}}{\partial x}+\bar{V} \frac{\partial \bar{V}}{\partial y}+\frac{\partial \bar{v}^{2}}{\partial y}+\frac{\partial \overline{u v}}{\partial x}=-\frac{1}{\rho} \frac{\partial \bar{P}}{\partial y}+\nu\left(\frac{\partial^{2} \bar{V}}{\partial x^{2}}+\frac{\partial^{2} \bar{V}}{\partial y^{2}}\right)
\end{aligned}
$$

The equation of continuity read's

$$
\frac{\partial \bar{U}}{\partial x}+\frac{\partial \bar{V}}{\partial y}=0
$$

Eq.(A-2) can be simplified as follows by neglecting $y$ direction mean velocity $\bar{V}$ and the fourth term on the lefthand side in Eq.(A-2)

$$
\frac{\partial \bar{v}^{2}}{\partial y}=-\frac{1}{\rho} \frac{\partial \bar{P}}{\partial y}
$$

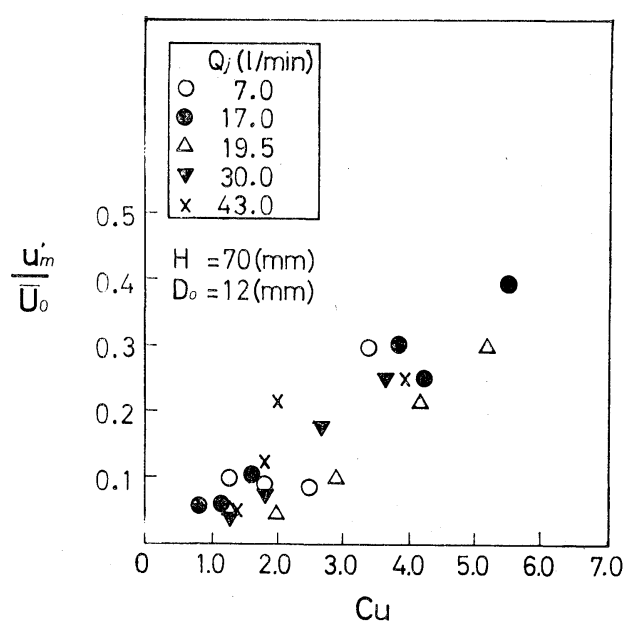

Fig. 11 Relative turbulence intensity averaged in $y$-direction at the position of maximum recirculation

If the turbulence intensity in the ambient flow is neglected compared with the turbulence intensity in jet mixing region, the above equation can be written

$$
\bar{P}+\rho \bar{v}^{2}=\bar{P} a
$$

1. The equation of motion of ambient flow

The ambient flow is assumed to be parallel frictionless flow and to be not turbulent.

Hence

$$
\begin{aligned}
& \overline{u v}=0 \quad \bar{u}^{2}=0 \\
& \bar{V} \frac{\partial \bar{U}}{\partial y}=0
\end{aligned}
$$

Then Eq. (A-1) reduces to

Hence

$$
\bar{U}_{a} \frac{\partial \bar{U}_{a}}{\partial x}=-\frac{1}{\rho} \frac{\partial \bar{P}}{\partial x}
$$

$$
\frac{1}{2} \rho \bar{U}_{a}^{2}+\bar{P}_{a}=\bar{P}_{t}=\text { const. }
$$

2. The equation of motion of jet

Eq.(A-4) being substituted into Eq. (A-1) and $\bar{V}=0$ being assumed, the equation of motion on the center line of jet is obtained as

$$
\bar{U}_{0} \frac{\partial \bar{U}_{0}}{\partial x}+\frac{\partial\left(\bar{u}^{2}-\bar{v}^{2}\right)}{\partial x}=-\left.\frac{\partial \overline{u v}}{\partial y}\right|_{y=0}-\frac{1}{\rho} \frac{\partial \bar{P}_{a}}{\partial x}
$$

in which the molecular viscosity term was neglected, compared with the turbulent shear stress term.

3. The equation of total momentum flux

Total momentum flux through a cross-section of vessel is constant. By substituting Eq.(A-3) and Eq.(A-4) into Eq.(A-1) and integrating, one gets

$$
\int_{-h}^{h}\left[\bar{U}^{2}+\left(\bar{u}^{2}-\bar{v}^{2}\right)\right] d y+\frac{2 h}{\rho} \bar{P}_{a}=\frac{M}{\rho}=\text { const. }
$$

4. The equation of total mass flux

Total mass flux through a cross-section of vessel is constant.

\section{(B) Eddy viscosity}

$$
\int_{-h}^{h} \bar{U} d y=Q=\text { const. }
$$

H. Goertler showed the virtual kinematic viscosity for two-dimensional free turbulent jet by the following expression (see Ref. 11)

$$
\varepsilon=\kappa b_{1 / 2} \bar{U}_{0}
$$

where $\bar{U}_{0}$ denotes the fluid velocity on the center line, $b_{1 / 2}$ is the lateral distance from the center line of the jet where the velocity is just half of the velocity on the center line, and 
$\kappa$ is an empirical constant. The value of $\kappa$ was determined experimentally by H. Reichard ${ }^{11}$.

Applying the above expression to the confined jet, and replacing the center line velocity $\bar{U}_{0}$ with the relative velocity $\bar{W}_{0}$, one gets

$$
\varepsilon=2 \sigma l \bar{W}_{0}
$$

where $l$ is the effective width of jet and can be expressed by $b_{1 / 2}$ as

then

$$
l=1.06 b_{1 / 2}
$$

$$
\sigma=0.0175
$$

(C) The starting position of recirculation

Eq.(A-8) can be expressed as follows by effective width of jet

$$
\begin{aligned}
\int_{-h}^{h} \bar{U} d y & =\int_{-h}^{h}\left(\bar{W}+\bar{U}_{a}\right) d y \\
& =2 \bar{W}_{0} l+2 \bar{U}_{a} h \\
& =Q
\end{aligned}
$$

This equation reduces to

$$
R+\frac{2 \vec{U}_{a} h}{Q}=1
$$

Then, the starting position of recirculation $\left(\bar{U}_{a}=0\right)$ is expressed by the position where $R=1$.

\section{Nomenclature}

$b_{1 / 2}=$ distance from the axis of jet where $\bar{U}=0.5 \bar{U}_{0}$

$C_{u}=$ Curtet number

[cm]

$d=$ inner diameter of jet nozzle

$D_{0}=$ outer diameter of jet nozzle

$[-]$

$[\mathrm{cm}]$

$E_{1}(f)=$ energy spectrum function of turbulence velocity $u$

$f=$ frequency of turbulence velocity

$\left[\mathrm{cm}^{2} / \mathrm{sec}^{2}\right]$

$[\mathrm{HZ}]$

$[\mathrm{cm}]$

[mm]

$H=$ half width of duct

$K=$ momentum coefficient

$l=$ effective width of jet

$M=$ total momentum flux

$\bar{P} \quad=$ static pressure in the jet mixing zone

$\bar{P}_{a}=$ static pressure in the ambient flow zone

$\bar{P}_{t}=$ total pressure in the ambient flow zone

$q=$ excess flow rate

$Q=$ total flow rate

$Q_{a}=$ ambient flow rate

$Q_{j}=$ jet flow rate

$\left[\mathrm{g} / \mathrm{cm} \cdot \mathrm{sec}^{2}\right.$

$\left[\mathrm{g} / \mathrm{cm} \cdot \mathrm{sec}^{2}\right]$

$\left[\mathrm{g} / \mathrm{cm} \cdot \mathrm{sec}^{2}\right]$

$\left[\mathrm{g} / \mathrm{cm} \cdot \mathrm{sec}^{2}\right]$

$[\mathrm{cc} / \mathrm{sec}]$

$[\mathrm{cc} / \mathrm{sec}]$

$[\mathrm{cc} / \mathrm{sec}]$

$[\mathrm{cc} / \mathrm{sec}]$
$R=$ dimensionless excess flow rate $(=q / Q)$

$R_{e d}=$ Reynolds number $\left(=\bar{U}_{n} d / \nu\right)$

$u=$ turbulence component in $x$-directicn

$u^{\prime}=$ turbulence intensity $\left(=\sqrt{\bar{u}^{2}}\right)$

$u^{\prime}=$ turbulence intensity averaged in $y$-direction \begin{tabular}{l}
$\left(=\frac{1}{h} \int_{0}^{h} u^{\prime} d y\right) \quad[\mathrm{cm} / \mathrm{sec}]$ \\
\hline
\end{tabular}

$\bar{U}=$ time mean velocity in $x$-direction $\quad[\mathrm{cm} / \mathrm{sec}]$

$\bar{U}_{a}=$ time mean velocity of ambient flow $[\mathrm{cm} / \mathrm{sec}]$

$\bar{U}_{n}=$ time mean velocity of jet at the exit of nozzle

$\bar{U}_{0}=$ time mean velocity at $y=0$

$v=$ turbulence component in $y$-direction

$v^{\prime}=$ turbulence intensity $\left(=\sqrt{\overline{\bar{v}}^{2}}\right)$

$\bar{V} \quad=$ time mean velocity in $y$-direction

$\bar{W} \quad=$ relative velocity in $x$-direction

$\bar{W}_{0}=$ relative velocity at $y=0$

$x=$ Cartesian coordinate

$X=$ dimensionless coordinate $(=\sigma x / h)$

$y=$ Cartesian coordinate

$=$ eddy viscosity

$=$ empirical constant of eddy viscosity

$=$ effective width ratio $(=l / h)$

$=$ kinematic viscosity

$=3.1415$

$=$ density of fluid

$=$ empirical constant of eddy viscosity

$[\mathrm{cm} / \mathrm{sec}]$

$[\mathrm{cm} / \mathrm{sec}]$

$[\mathrm{cm} / \mathrm{sec}]$

$[\mathrm{cm} / \mathrm{sec}]$

$[\mathrm{cm} / \mathrm{sec}]$

$[\mathrm{cm} / \mathrm{sec}]$

[cm]

$[-]$

$[\mathrm{cm}]$

$[\mathrm{cm} / \mathrm{sec}]$

$[\mathrm{cm} / \mathrm{sec}]$

$\left[\mathrm{g} / \mathrm{cm}^{3}\right]$

\section{Literature Cited}

1) Becker, H. A., H. C. Hottel and G. C. Williams: Ninth Symposium on Combustion, p. 1 (1962)

2) Becker, H. A., H. C. Hottel and G. C. Williams: Tenth Symposium on Combustion, p. 1253 (1964)

3) Beer, J. M.; J. Inst. Fuel, 37, 286 (1964)

4) Chedaille, J., W. Leuckel and A. K. Chesters: J. Inst. Fuel, 39, 506 (1966)

5) Curtet, R.: Combust. Flame, 2, 383 (1958)

6) Hedley, A. B. and E. W. Jackson: J. Inst. Fuel, 39, 208 (1966)

7) Hinze, J. O.: “Turbulence", p. 438 (1959)
8) Hubburd, E. H.: J. Inst. Fuel, 35, 160 (1962)

9) Okita, N. and S. Ito: Kagaku Kōgaku, 30, 934 (1966)

10) Thring, M. W. and M. P. Newby: Fourth Symposium on Combustion, p. 789 (1953)

11) Schlichting, H: "Boundary Layer Theory", p. 605(1960)

12) Wygnanski, I. and H. E. Fiedler: J. Fluid Mech., 38, 577 (1969)

13) Wygnanski, I. and H. E. Fiedler: J. Fluid Mech., 41, 327 (1970) 